\title{
Mass Footprints of the North Pacific Atmospheric Blocking Highs
}

2

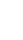

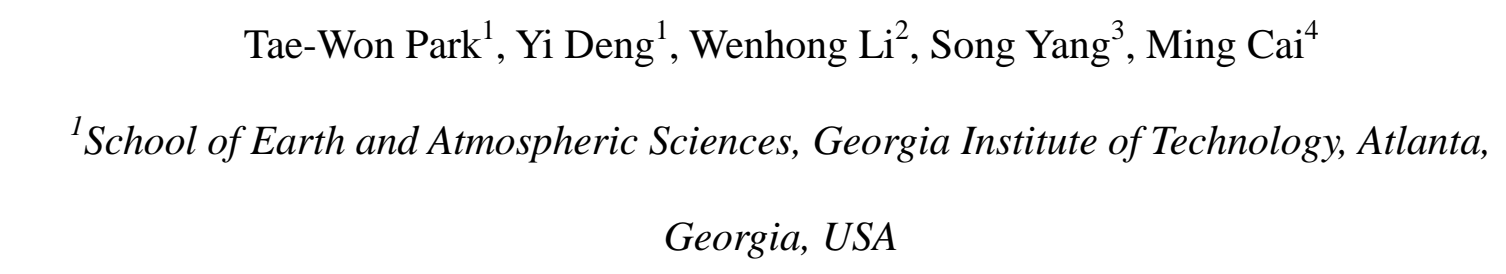

${ }^{2}$ Earth and Ocean Sciences, Nicholas School, Duke University, Durham, North Carolina,

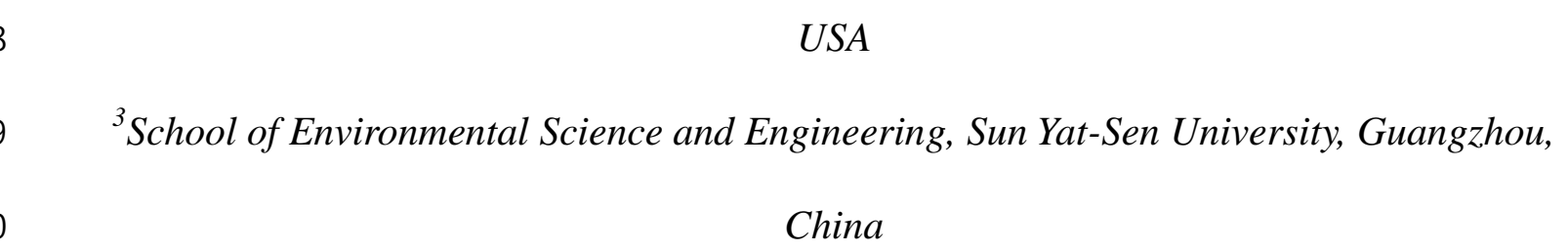

${ }^{3}$ School of Environmental Science and Engineering, Sun Yat-Sen University, Guangzhou,

Submitted to Journal of Climate

August 2014

Corresponding author address:

Tae-Won Park, School of Earth and Atmospheric Sciences, Georgia Institute of Technology, Atlanta, Georgia. E-mail: taewon.park@eas.gatech.edu Yi Deng, School of Earth and Atmospheric Sciences, Georgia Institute of Technology, Atlanta, Georgia. E-mail: yi.deng@eas.gatech.edu 


\section{Abstract}

The mass footprints associated with atmospheric blocks over the North Pacific are evaluated by constructing daily tendencies of total mass over the blocking domain from 3dimensional mass fluxes throughout the lifecycle of a composite blocking event. The results highlight the major role of mass convergence driven by low-frequency (with periods > one week) atmospheric motions (disturbances) during both the development and decay stage of a block. Specifically, low-frequency motions are responsible for the accelerated mass build-up 3 days prior to the peak intensity of a block and they also account for the rapid mass loss afterwards. High-frequency motions related to sub-weekly-scale disturbances have statistically significant contributions to the mass loss during the decay stage, and also show positive yet very weak contributions to the development of the blocking high at the very early stage of the event. Majority of the mass convergence/divergence responsible for the intensification/decay of the blocking high occurs in the mid-upper troposphere and is largely attributed to mass flux driven by meridional winds. Also discussed are the implications of this new mass perspective of atmospheric blocks for understanding dynamics of blocking highs and for model bias detection and attribution.

40 


\section{Introduction}

Blocking highs constitute a crucial component of the extratropical atmospheric lowfrequency variability. The flow fields of these "atmospheric blocks" are characterized by persistent anticyclones that that tend to obstruct the normal zonal flow (Berggren et al. 1949; Rex 1950). Being vertically coherent and quasi-stationary, blocking highs exert strong impacts not only on regional weather patterns but also on global atmospheric circulation (Carrera et al. 2004; Trigo et al. 2004).

On one hand, high-frequency (sub-weekly-scale) transient eddies have been known to play a critical role in the development of atmospheric blocks. For example, Green (1977) suggested the importance of storm track eddies in maintaining blocking flows. Nakamura and Wallace (1993) provided further evidences of elevated activity of high-frequency baroclinic eddies prior to the onset of blocking events. Blocking formation in some general circulation models (GCMs) was also shown to be tied to dynamical forcing of high-frequency eddies (Maeda et al. 2000; Shutts 1983). On the other hand, effects of low-frequency (10-30 day period) eddies themselves are also felt throughout the lifecycles of atmospheric blocks. For example, the occurrence of a quasi-stationary wave train may induce the formation of a persistent anticyclonic flow anomaly (Stewart 1993). Colucci (1985) showed that the planetary wave environment created by a vorticity source during explosive cyclogenesis can result in subsequent downstream blocking. Breaking of low-frequency Rossby waves entails reversals of the meridional gradients of potential temperature at the dynamical tropopause, which are also recognized as key features of atmospheric blocks (Pelly and Hoskins 2003). Nakamura et al. (1997) pointed out that forcing from high-frequency eddies is indispensable for blocking formation over the North Pacific while low-frequency dynamics represented by the emergence of a quasi-stationary Rossby wave train over the North Atlantic is more important for blocking development over Europe. 
From the point of view of mass change, the formation of a blocking high can be regarded as the process of mass convergence into the air column underneath which the surface high emerges. In the literature, however, no study has examined the development and decay of blocking highs from the perspective of mass accumulation and dissipation. Homeyer and Bowman (2013) investigated the mass transport between tropical and extratropical regions associated with events of Rossby wave breaking through Lagrangian trajectory calculations, but "area" transport" instead of actual mass was dealt in the analysis. The goal of this study is to provide a preliminary, mass-circulation-based assessment of the relative roles of atmospheric eddies (motions) of different temporal scales in determining the lifecycle of atmospheric blocks observed over the North Pacific. The calculations will be done in a semiLagrangian framework and focus on quantifying the column mass divergence/convergence based upon daily 3-dimensional (3D) mass flux in isentropic coordinates.

\section{Methods}

The study period considered here is 1979 to 2010 . The data used in the analysis are obtained from the European Center for Medium-Range Weather Forecasts (ECMWF) ReAnalysis Interim (ERA-Interim) (Dee et al. 2011). Specifically, daily potential temperature $(\theta)$ on the 2-potential-vorticity-unit (PVU) surface is used to identify prominent blocking events. Mass fluxes across boundaries of blocking events are derived from daily geopotential height $(\mathrm{Z})$, temperature $(\mathrm{T})$, zonal and meridional winds ( $\mathrm{u}$ and $\mathrm{v})$, and vertical velocity $(\omega)$ on 36 isentropic levels, which are interpolated from 37 isobaric levels in the original ERAInterim data.

The detection of blocking events follows the methodology of Pelly and Hoskins (2003). An instantaneous (daily) blocking index is first calculated for each longitude centered about a line of central blocking latitude that roughly follows the line of local maxima in the kinetic 
energy of atmospheric transients. A blocking event is considered occurring if the blocking index is positive. To identify prominent, long-lasting blocking events, we adopt the criteria that the instantaneous blocking (positive block index values) should occur over at least $10^{\circ}$ of longitude and last at least 4 consecutive days. Focusing on a region extending from the eastern North Pacific to the west coast of North America, we are able to detect a total of 30 major blocking events for the study period and these events are used in the subsequent composite analysis.

The calculation of mass flux is done for a cuboid bounded by two adjacent isentropic levels in the vertical and with $60^{\circ}$ (longitude) and $20^{\circ}$ (latitude) in length in the horizontal. The center of the cuboid is placed at the location of the maximum surface pressure that is identified for each selected blocking event when it attains its peak intensity (defined as "day 0 " in the analysis). In an isentropic layer bounded two adjacent isentropic levels, atmospheric mass per unit area (unit: $\mathrm{kg} \cdot \mathrm{m}^{-2}$ ) is computed following

$$
M=-\frac{1}{g} \cdot \Delta p
$$

where $g$ is gravity constant $\left(9.81 \mathrm{~ms}^{-2}\right)$ and $\Delta p$ is the pressure difference between the two levels. The net convergence of mass into the region of a blocking high is the sum of 3 components: the convergence in the zonal, meridional, and vertical direction. Zonal mass convergence can be estimated as the difference of the total zonal mass fluxes $(m f x)$ between the western and eastern boundary of the analysis region following

$$
m c_{z o n a l}=\sum_{w b} m f x_{w b}-\sum_{e b} m f x_{e b}=\sum_{w b} M_{w b} \cdot a \cdot \Delta \phi \cdot u_{w b}-\sum_{e b} M_{e b} \cdot a \cdot \Delta \phi \cdot u_{e b},
$$

where $w b$ and $e b$ stand for "western boundary" and "eastern boundary", respectively; $a$ is the mean radius of the earth $(6370 \mathrm{~m}) ; \Delta \phi$ is the latitude interval $\left(1.5^{\circ}\right) ; u$ is zonal wind. In a similar manner, meridional mass convergence is computed as the difference of the 
total meridional mass fluxes ( $m f y$ ) between the southern and northern boundary of the analysis region following

$$
m c_{\text {meridional }}=\sum_{s b} m f y_{s b}-\sum_{n b} m f y_{n b}=\sum_{s b} M_{s b} \cdot a \cdot \cos \phi \cdot \Delta \lambda \cdot v_{s b}-\sum_{n b} M_{n b} \cdot a \cdot \cos \phi \cdot \Delta \lambda \cdot v_{n b},
$$

where $s b$ and $n b$ standard for "southern boundary" and "northern boundary", respectively; $\Delta \lambda$ is the longitude interval $\left(1.5^{\circ}\right) ; \quad v$ is meridional wind.

Vertical mass convergence into the cuboid is calculated as the difference between the mass flux ( $m f z)$ at the upper and lower isentropic level bounding the cuboid following

$$
m c_{\text {vertical }}=\sum_{l b} m f z_{l b}-\sum_{u b} m f z_{u b}=\sum_{l b} M_{l b} \cdot A \cdot \frac{\dot{\theta_{l b}}}{\Delta \theta_{l b}}-\sum_{u b} M_{u b} \cdot A \cdot \frac{\dot{\theta_{u b}}}{\Delta \theta_{u b}},
$$

where $l b$ and $u b$ stand for "lower boundary" and "upper boundary", respectively. $A$ is the

area of the grid box and $\dot{\theta}$ is the total diabatic heating rate, which is estimated through the thermodynamic energy equation.

To investigate the contributions of atmospheric eddies of different frequencies to the total mass flux convergence, we further decompose the mass flux across the horizontal and vertical boundaries of the analysis region into those associated with wind fluctuations at various frequencies. Specifically, we separate the total winds into a high-frequency component (obtained via 6-day high-pass filter) and a low-frequency/stationary component (obtained as the difference between the total and high-pass filtered value). This decomposition produces 3 parts in the mass flux: (i) flux due to high-frequency eddies, (ii) flux due to lowfrequency/stationary eddies, and (iii) flux due to the interaction between high- and lowfrequency/stationary eddies following

$$
M \cdot V=\left(M_{\text {high }}+M_{\text {low }}\right) \cdot\left(V_{\text {high }}+V_{\text {low }}\right)=\underbrace{M_{\text {high }} \cdot V_{\text {high }}}_{(i)}+\underbrace{M_{\text {low }} \cdot V_{\text {low }}}_{(i i)}+\underbrace{M_{\text {high }} \cdot V_{\text {low }}+M_{\text {low }} \cdot V_{\text {high }}}_{\text {(iii) }} .
$$




\section{Results}

Figure 1a shows the composite mean $300 \mathrm{hPa}$ geopotential height (contours) and the corresponding anomaly field (shading) for the identified 30 North Pacific blocking events at the time of peak intensity (day 0 ). The positive height anomaly characterizing the blocking sits over the southern Alaska with local maximum values reaching 1,500 $\mathrm{m}^{2} \mathrm{~s}^{-2}$. A weaker negative height anomaly is found southwest of the blocking high. Intrusion of high- $\theta$ (potential temperature) airmass into the high latitudes is clearly visible through the folding of the $326 \mathrm{~K} \theta$ contour (think solid line in Fig. 1a). Since the poleward extrusion of high- $\theta$ air is more pronounced compared to the equatorward extrusion of low- $\theta$ air, the composite blocking represents dynamically a warm-cyclonic Rossby wave breaking event (Masato et al. 2012). Figure $1 \mathrm{~b}$ plots the temporal evolution of the airmass above $280 \mathrm{~K} \theta$ surface over a blocking area $\left( \pm 30^{\circ}\right.$ longitude and $\pm 10^{\circ}$ latitude from the center of each blocking event) throughout the lifecycle of the composite blocking event (day -8 to day +8 ). Note that the $280 \mathrm{~K}$ surface is used here instead of the actual surface to avoid the relatively large errors produced when near surface data is interpolated from isobaric to isentropic levels (Ziv and Alpert 1994). Furthermore, the airmass above $280 \mathrm{~K}$ in the study region in the lifecycle of the blocking events on average accounts for $97 \%$ of the total mass in a column, and this allows our calculations to capture majority of the processes responsible for the mass accumulation and dissipation during the lifecycle of the blocking events. Figure $1 \mathrm{~b}$ shows that the mass in the blocking domain steadily increases starting from day -8 and the increase accelerates on day -3 . The mass reaches its peak value around day +1 , slightly later compared to the time when the composite height anomaly attains its maximum value (day 0). The decay of the block is characterized by a rather rapid dissipation of mass out of the domain. Approximately 3 days after the peak intensity of the blocking, the total mass in the domain goes back to the 
pre-blocking level.

The accumulation and dissipation of airmass over the blocking domain is further illustrated by the day-to-day mass tendency shown in Figure 2a (solid line). Positive mass tendency lasts throughout the period day -8 to day +1 , responsible for the eventual mass build-up while large amplitude, negative tendency dominates since day +2 , indicating a rapid evacuation of mass out of the domain. Shaded bars in Figure 2a are the mass tendencies estimated through the total mass convergence (into the blocking domain) computed on isentropic surfaces with isentropic wind and pressure data. The daily mass convergence matches the daily mass tendency (derived from pressure change) very well throughout the lifecycle of the blocking event. Following Eq. (5), we decompose the total daily mass convergence into components associated with high frequency atmospheric motion (Fig. 2b), low-frequency motion (Fig. 2d) and the interaction between high- and low-frequency motions (Fig. 2c). It is quite evident that the mass convergence driven by low-frequency motions (Fig. 2d) dictates the mass tendency during both the development and decay stage of the blocking event. High-frequency, synoptic-scale motions (Fig. 2b), on one hand, tend to work against the low-frequency motions by producing weak yet statistically significant mass depletion during the development stage of the blocking (day -1 to day +1 ). On the other hand, mass depletion driven by high-frequency motions during the period day +2 to day +3 contributes positively to the decay of the blocking. It is also interesting to note that during the early stage of the blocking development, e.g., day -8 to day -4 , high-frequency motions have consistent positive contributions. This is in agreement with earlier studies that demonstrated the importance of high-frequency disturbances in the early development of atmospheric blocks. It also suggests that dynamical effects (i.e., heat and momentum fluxes) of high-frequency disturbances on blocking development are more evident in fields such as middle/upper level geopotential height while mass footprints of atmospheric blocks are less influenced directly 
by high-frequency disturbances. The magnitude of the mass convergence associated with the interaction between high- and low-frequency motions (Fig. 2c) is also small compared to the low-frequency motions and it has a negative contribution to the blocking decay on day +3 . Figure 2 thus proves that the mass footprints of a Pacific blocking event, i.e., the build-up and decay of the surface high, is mainly driven by atmospheric motions with a period longer than a week.

We further evaluate the vertical structure of the mass convergence during the lifecycle of a blocking event. Figure 3a plots the mass tendency derived from isentropic level pressure change computed for individual isentropic layers between $280 \mathrm{~K}$ and $370 \mathrm{~K}$ from day -8 to day +8 . It agrees very well with the mass convergence shown in Figure $3 b$. Prior to day +1 , the mass accumulation over the blocking domain is largely caused by mass build-up between $300 \mathrm{~K}$ and $340 \mathrm{~K}$ in the upper troposphere. This build-up of mass is accompanied by weak mass loss underneath $300 \mathrm{~K}$. During the decaying stage of the blocking (after day +1 ), the mass loss in the column also occurs primarily in the upper troposphere while weak mass build-up is found underneath $290 \mathrm{~K}$. The vertical structure of mass convergence is further divided into two parts: one driven horizontal motions (Fig. 3c) and the other by vertical motions (Fig. 3d). By definition, the sum of the mass convergence associated with vertical motions in the vertical direction reduces to the weak vertical mass flux at $280 \mathrm{~K}$, the lower boundary considered in the analysis. This is clearly the case given the significant cancellations between mass divergence $(-)$ and convergence $(+)$ in the vertical direction seen in Figure 3d. Mass convergence associated with horizontal motions (Fig. 3c) drives the mass change in the blocking domain and its structure bears a high degree of similarity with that of the total mass tendency (Fig. 3a). A further separation of the horizontal mass convergence into the components related to zonal (Fig. 3e) and meridional (Fig. 3f) transport reveals the dominant role of meridional transport in determining the mass footprints of an atmospheric 
block. The magnitude of the meridional transport is about 2-3 times greater than that of the zonal transport. Specifically, the meridional transport is responsible for the accelerated mass build-up starting from day -2 and the rapid mass loss starting from day +2 (Fig. 3f). The statistically significant signals of transport are found between $300 \mathrm{~K}$ and $320 \mathrm{~K}$ during the development stage of the blocking and between $320 \mathrm{~K}$ and $340 \mathrm{~K}$ during the decay stage of the blocking. The zonal transport (Fig. 3e) generally works against the meridional transport during the development stage of the blocking and contributes positively to the decay in the column integrated sense.

Figure 4 shows the breakdown of the Figure 3 results into components associated with highfrequency and low-frequency motions. Consistent with the previous discussion, lowfrequency motions prove to be the main driver of the mass footprints of the blocking event (i.e., comparing Fig. $4 b$ with Fig. 3b), although high-frequency motions show some positive contributions to the mass build-up prior to day -3 . This is also true in terms of the mass convergence associated with horizontal motions (i.e., comparing Fig. 4d with Fig. 3c). Similarly, low-frequency motions determine the structures of mass convergence driven by both zonal (Fig. 4f versus Fig. 3e, Fig. 4h versus Fig. 3f) and meridional transport. Note that the high- and low-frequency interaction terms are omitted here due to the lack of statistical significance.

\section{Concluding Remarks} investigated based on the evaluation of the 3D mass fluxes and the resulted mass convergence into the blocking domain. The results highlight the critical role of mass convergence driven by low-frequency atmospheric motions in the development and decay of a blocking event. 
mass build-up 3 days prior to the peak intensity of a block and they also account for the rapid mass loss during the decay stage of the block. High-frequency motions have statistically significant contributions to the mass loss during the decay stage, and also show positive yet very weak contributions to the development of the blocking high at the very early stage of the event. This later result is consistent with previous findings that suggested a "precursor" role of high-frequency, synoptic-scale disturbances in the development of atmospheric blocks. It is also found that majority of the mass convergence/divergence responsible for the intensification/decay of the blocking high occurs in the mid-upper troposphere and is largely attributed to meridional mass transport, i.e., mass flux related to meridional winds. Mass flux related to zonal winds has positive contributions to the decay of the blocking. In summary, low-frequency atmospheric motions in the meridional direction largely determine the mass footprints of atmospheric blocks and high-frequency motions play an auxiliary role mostly during the decay stage.

The present analysis is among the earliest to approach the blocking mechanism from a mass circulation perspective. It offers a different way to understand the lifecycle characteristics of atmospheric blocks by focusing on their surface/near-surface signatures, i.e., blocking highs. This is in contrast to traditional analyses that tend to focus on mid-upper level height or PV anomalies. This new perspective also provides a different angle to detect and trace potential model biases in simulating atmospheric blocks. Additionally, the calculations of 3D mass flux can facilitate the diagnosis of transport properties of blocking events and can be readily extended to the analysis of other classes of weather phenomena such as extratropical cyclones and hurricanes. A notable limitation of the current approach is the use of a fixed-size blocking domain since in reality flow deformation (e.g., zonal stretching and meridional compression) tends to occur during the decay stage of a blocking event (Carrera et al. 2004). Further improvement of the diagnosis approach will be explored when we continue 
the examination of atmospheric blocks over other geographical domains such as the North Atlantic.

\section{Acknowledgments.}

The ERA-Interim data used in this study were provided by the European Centre $\mathrm{f}$ or Medium-Range Weather Forecast (ECMWF). This study is supported by the National $\mathrm{S}$ cience Foundation through the grants AGS-1147601 and AGS-1354402.

\section{References}

Berggren, R., B. Bolin, and C. G. Rossby, 1949: An Aerological Study of Zonal Motion, its Perturbations and Break-down. Tellus, 1, 14-37.

Carrera, M. L., R. W. Higgins, and V. E. Kousky, 2004: Downstream weather impacts associated with atmospheric blocking over the northeast Pacific. J Climate, 17, 4823-4839. Colucci, S. J., 1985: Explosive Cyclogenesis and Large-Scale Circulation Changes Implications for Atmospheric Blocking. J Atmos Sci, 42, 2701-2717.

Dee, D. P., and Coauthors, 2011: The ERA-Interim reanalysis: configuration and performance of the data assimilation system. Q J Roy Meteor Soc, 137, 553-597.

Green, J. S. A., 1977: The Weather during July 1976: Some Dynamical Considerations of the Drought. Weather, 32, 120-126.

Homeyer, C. R., and K. P. Bowman, 2013: Rossby Wave Breaking and Transport between the Tropics and Extratropics above the Subtropical Jet. J Atmos Sci, 70, 607-626.

Maeda, S., C. Kobayashi, K. Takano, and T. Tsuyuki, 2000: Relationship between singular modes of blocking flow and high-frequency eddies. J Meteorol Soc Jpn, 78, 631-646.

Masato, G., B. J. Hoskins, and T. J. Woollings, 2012: Wave-breaking characteristics of midlatitude blocking. Q J Roy Meteor Soc, 138, 1285-1296.

Nakamura, H., and J. M. Wallace, 1993: Synoptic Behavior of Baroclinic Eddies during the Blocking Onset. Mon Weather Rev, 121, 1892-1903.

Nakamura, H., M. Nakamura, and J. L. Anderson, 1997: The role of high- and low-frequency dynamics in blocking formation. Mon Weather Rev, 125, 2074-2093.

Pelly, J. L., and B. J. Hoskins, 2003: A new perspective on blocking. J Atmos Sci, 60, 743-755. 
291 Rex, D. F., 1950: Blocking Action in the Middle Troposphere and its Effect upon Regional 292 Climate. Tellus, 2, 196-211.

293 Shutts, G. J., 1983: The Propagation of Eddies in Diffluent Jetstreams - Eddy Vorticity 294 Forcing of Blocking Flow-Fields. Q J Roy Meteor Soc, 109, 737-761.

295 Stewart, D. A., 1993: Persistent Anomaly Forcing in a Two-Level Global Circulation Model. 296 J Atmos Sci, 50, 2710-2730.

297 Trigo, R. M., I. F. Trigo, C. C. DaCamara, and T. J. Osborn, 2004: Climate impact of the 298 European winter blocking episodes from the NCEP/NCAR Reanalyses. Clim Dynam, 23, 1729928.

300 Ziv, B., and P. Alpert, 1994: Isobaric to Isentropic Interpolation Errors and Implication to 301 Potential Vorticity Analysis. J Appl Meteorol, 33, 694-703. 


\section{List of Figures}

\section{North Pacific Blocking}
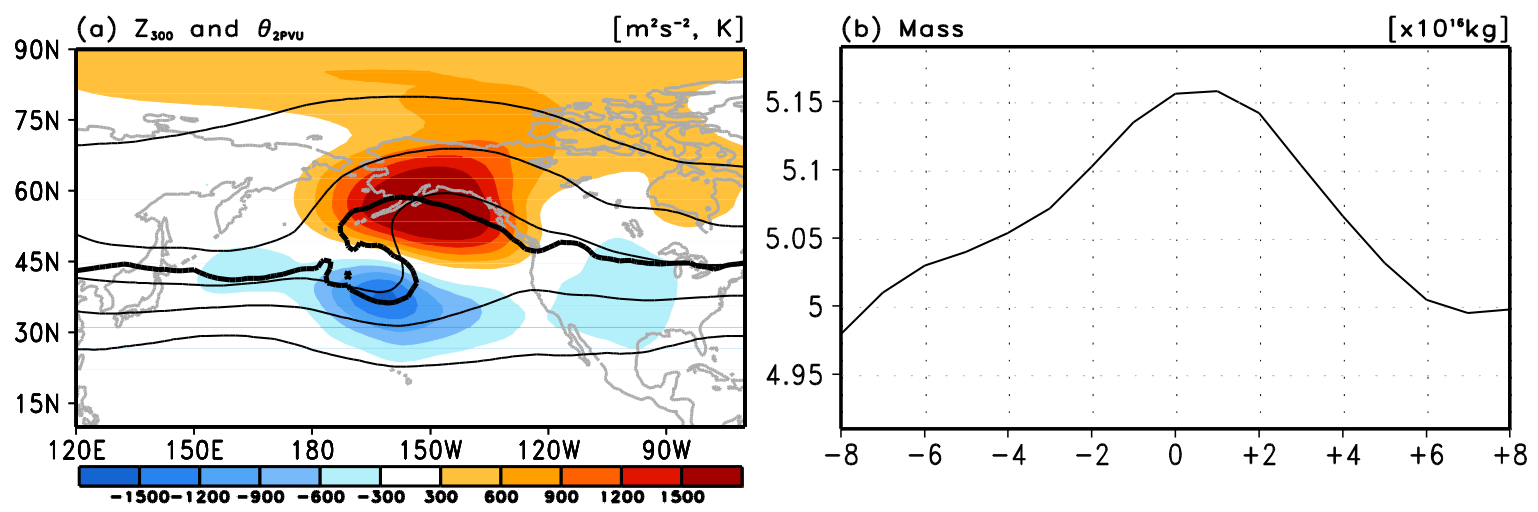

Figure 1. (a) The composite geopotential height at $300 \mathrm{hPa}$ (contour with an interval of $20 \mathrm{~m}^{2}$ $309 \mathrm{~s}^{-2}$ ) and the corresponding anomaly (shading) for the North Pacific blocking. The black solid 310 line indicates the $\theta$ contour of $326 \mathrm{~K}$ on PVU $=2$ surface.. (b) Temporal evolution of the total mass (unit: kg) within the blocking domain above $280 \mathrm{~K}$. 
(a) Mass Ten. and Total Mass Con. $\left[\times 10^{\circ} \mathrm{kg} / \mathrm{s}\right]$
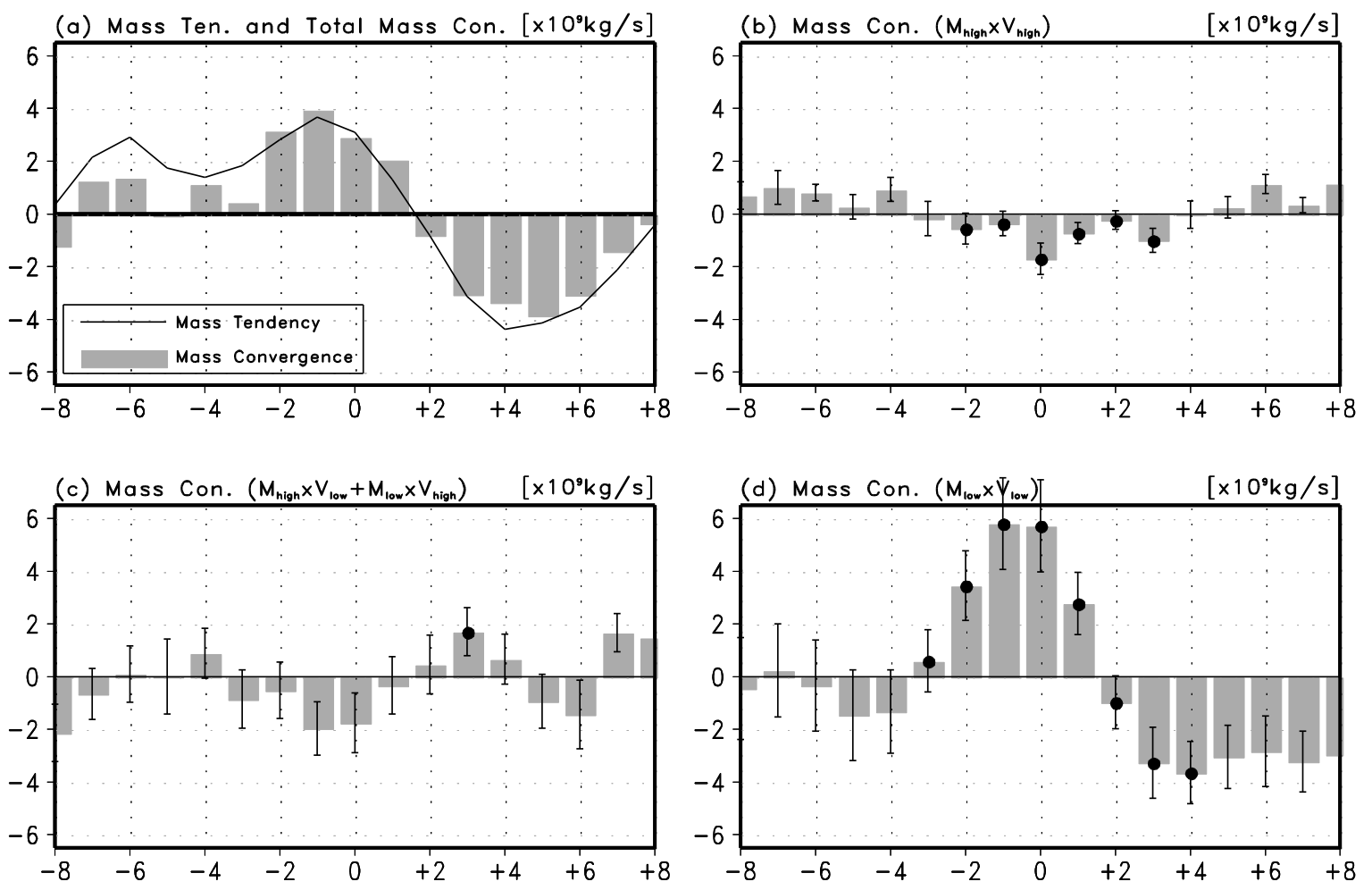

Figure 2. (a) Temporal evolutions of the daily mass tendency (solid line) and total mass convergence evaluated from 3D mass fluxes (shaded bars). Mass convergence driven by (b) high-frequency atmospheric motions, (c) the interaction between high- and low-frequency atmospheric motions, and (d) low-frequency motions (for detailed definitions, please see Section 2 of the main text). In (b)-(d), black dots indicate statistical significance at the $90 \%$ level and the error bars correspond to \pm 1 standard deviation error. Units: $\mathrm{kg} / \mathrm{s}$. 

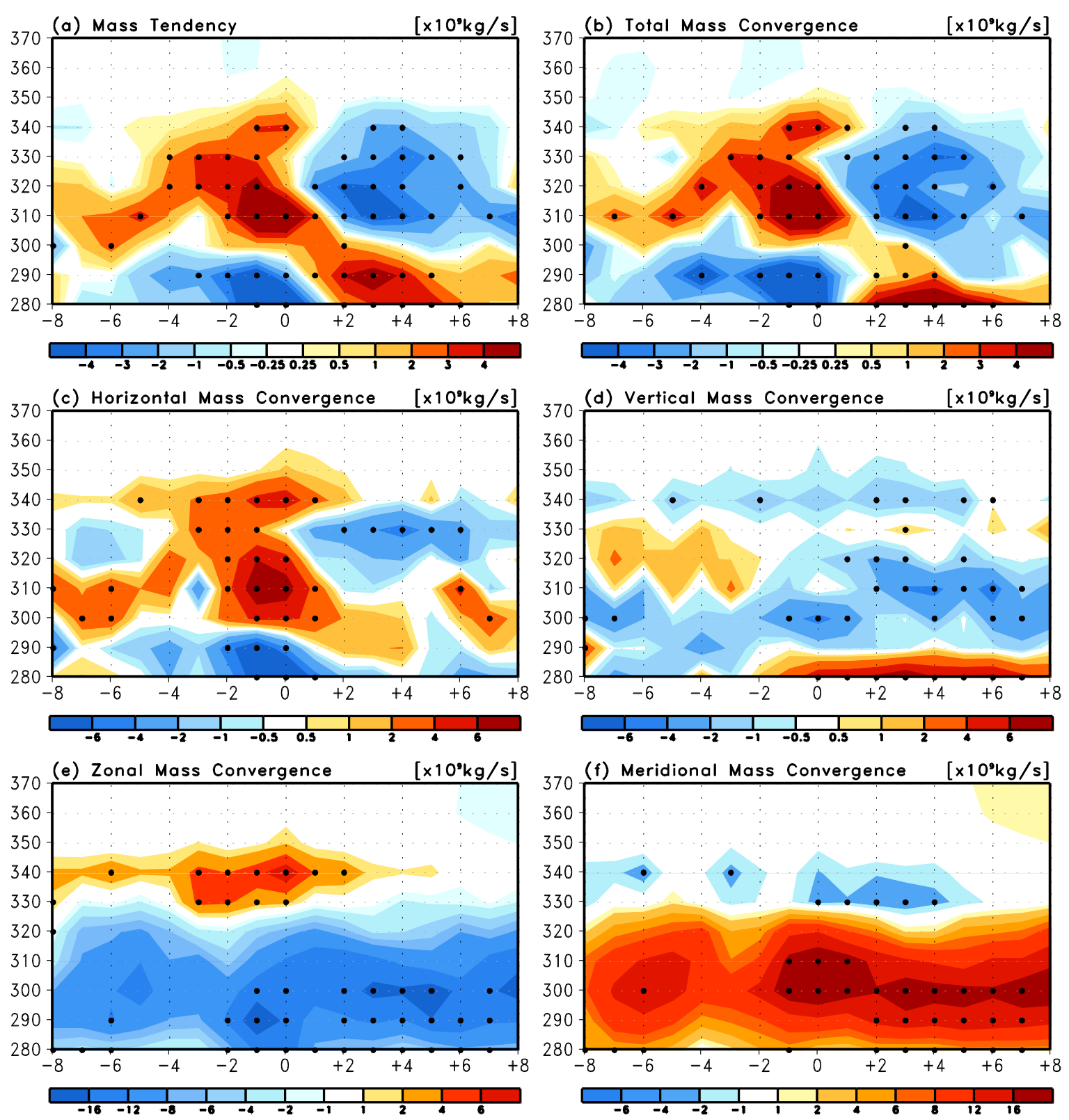

Figure 3. Temporal evolutions of (a) the mass tendency, and the mass convergence associated with (b) total, (c) horizontal, (d) vertical, (e) zonal, and (f) meridional transports for individual isentropic layers ranging from $280 \mathrm{~K}$ to $370 \mathrm{~K}$. Black dots indicate statistical significance at the $90 \%$ level. Units: $\mathrm{kg} / \mathrm{s}$. 

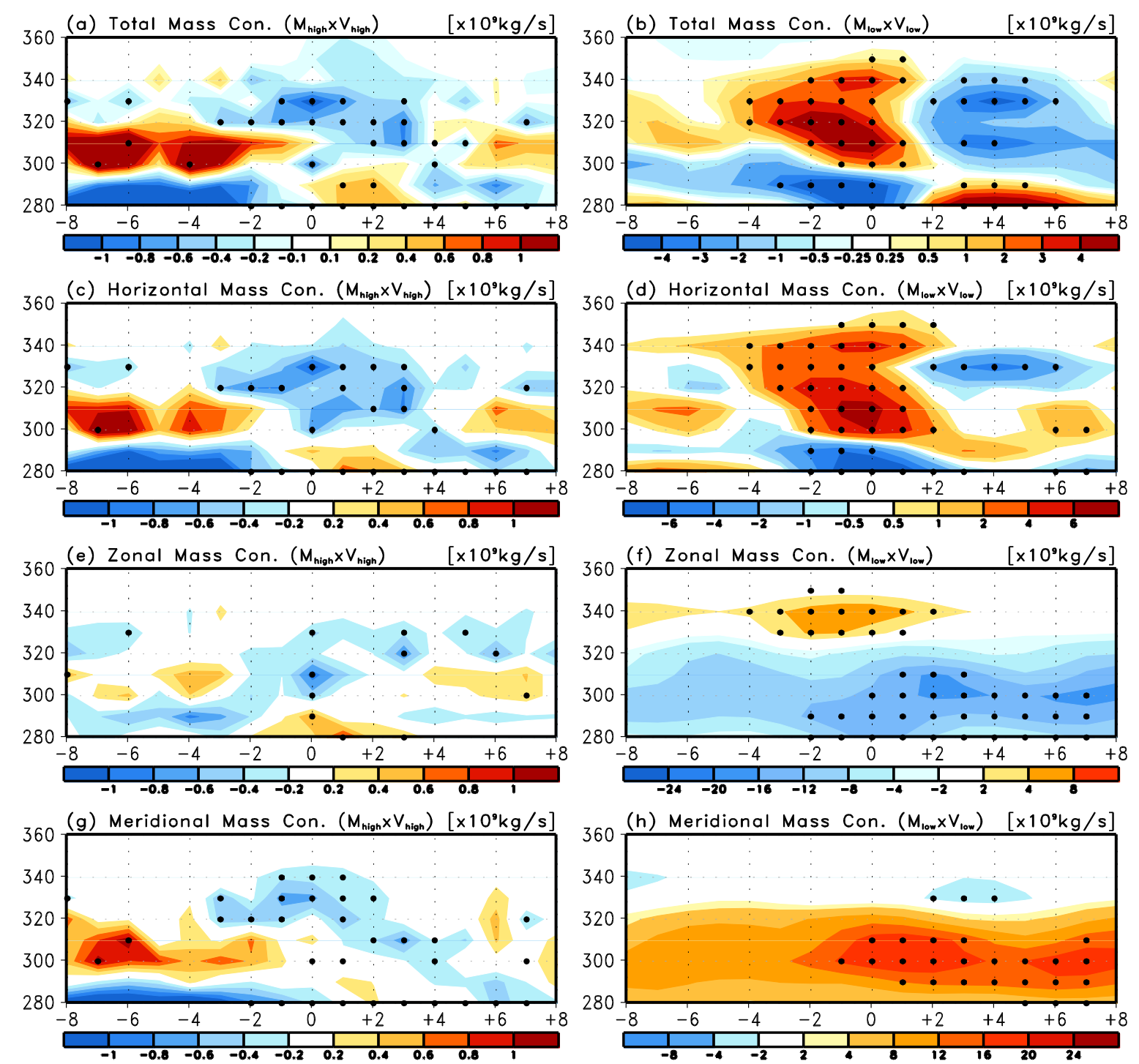

Figure 4. Temporal evolutions of the mass convergence associated with (a,b) total, $(\mathrm{c}, \mathrm{d})$, horizontal, $(\mathrm{e}, \mathrm{f})$ zonal, and $(\mathrm{g}, \mathrm{h})$ meridional transports for individual isentropic layers ranging from $280 \mathrm{~K}$ to $370 \mathrm{~K}$. All panels in the left column correspond to mass convergence driven by high-frequency motions and all panels in the right column correspond to mass convergence driven by low-frequency motions. Black dots indicate statistical significance at the $90 \%$ level.

332 Units: $\mathrm{kg} / \mathrm{s}$. 